01

\title{
Гибридный метод диабатизации и его применение к квазимолекуле СаH
}

\author{
(C) Д.В. Власов ${ }^{1}$, Д.С. Родионов ${ }^{2}$, А.К. Беляев ${ }^{2}$ \\ ${ }^{1}$ Т-Системс РУС, \\ 199034 Санкт-Петербург, Россия \\ ${ }^{2}$ Российский государственный педагогический университет им. А.И. Герцена, \\ 191186 Санкт-Петербург, Россия \\ e-mail: dmitry.vlasov@t-systems.com, dsrodionov@herzen.spb.ru, akbelyaev@herzen.spb.ru
}

Поступила в редакцию 05.10.2017 г.

В окончательной редакции 11.12.2017 г.

Предложена процедура диабатизации квантово-химических данных, позволяющая устранить проблему $\delta$-образных пиков матричных элементов неадиабатичности $\left\langle j\left|\frac{\partial}{\partial R}\right| k\right\rangle$ путем перехода к гибридному диабатическому представлению системы связанных уравнений ядерной динамики с использованием модельных матричных элементов неадиабатичности, приближающихся к изначально заданным. Результаты использования данного метода в рамках модели Ландау-Зинера представлены в работе на примере столкновительной системы $\mathrm{CaH}$.

DOI: $10.21883 /$ OS.2018.05.45935.221-17

\section{1. Введение}

Точность расчетов вероятностей переходов и сечений процессов при медленных атомных столкновениях оказывает существенное влияние при дальнейшем использовании полученных результатов в астрофизических приложениях. Отклонения от приближения локального термодинамического равновесия (не ЛТР эффекты) обусловливают необходимость последовательного квантового подхода к вычислению ядерной динамики атомных столкновительных систем. Особую роль при этом играют столкновения атомов металлов с атомами и ионами водорода, что обусловлено преобладанием таких процессов в условиях значительно меньшей концентрации электронов. Так, в атмосфере Солнца отношение концентрации электронов к концентрации атомов водорода составляет величину $\approx 10^{-4}[1]$. Таким образом, квантовый подход при расчете сечений процессов при атомных столкновениях дает существенный вклад в объяснение картины звездных спектров. Результаты таких вычислений используются в физико-химических расчетах статистического равновесия указанных сред. При этом обычно используют подход Борна-Оппенгеймера [2], который позволяет разделить электронные и ядерные движения в уравнении Шредингера рассматриваемой квазимолекулы. Решение электронной задачи проводится в рамках квантово-химических вычислений. Полученные квантово-химические данные используются при решении квантовой ядерной задачи $[3,4]$. Авторами был проведен ряд расчетов для таких систем как, например, $\mathrm{LiH}$ [5], $\mathrm{NaH}[6,7]$ и $\mathrm{MgH}$ [8]. Квантово-химические данные для этих квазимолекул использовались без изменений в рамках проведенных вычислений.

Настоящая работа затрагивает часть проблематики расчета квазимолекулы СаН. Интерес к этому типу столкновительной системы обусловлен тем, что кальций является $\alpha$-элементом [9], играющим важную роль при образовании сверхновых звезд. Как следствие, определение его концентрации вносит вклад в получение информации о звездной эволюции. Квантово-химические данные выбранной квазимолекулы имеют некоторые неудобные с точки зрения вычислений особенности. Конкретно, рассматривается близкое к сингулярному поведение матричных элементов неадиабатичности $\left\langle j\left|\frac{\partial}{\partial R}\right| k\right\rangle$ определенного типа и способ преодоления этого неудобства в рамках процедуры диабатизации квантово-химических данных. При этом рассматриваются только ковалентные ${ }^{2} \Sigma^{+}$-состояния квазимолекулы. Это обусловлено совпадением типа симметрии этих состояний с симметрией состояния ионной пары $\mathrm{Ca}^{+}\left(4 s^{2} S\right)+\mathrm{H}^{-}\left(1 s^{2}{ }^{1} S\right)$, значительным ионно-ковалентным взаимодействием на больших межъядерных расстояниях $[3,10,11]$ и преобладанием переходов между указанными ковалентными состояниями при малых энергиях столкновения.

Проведение расчетов атомных столкновительных систем в рамках квантовой ядерной задачи включает в себя выбор численных методов и подготовку квантовохимических данных. Первоначальный набор таких данных, как правило, задается в адиабатическом представлении, при котором матрица электронного гамильтониана имеет диагональный вид. Диагональные элементы этой матрицы представляют собой непересекающиеся потенциальные кривые для молекулярных состояний с одинаковой симметрией. В определенных областях межъядерных расстояний, где расщепления между указанными потенциалами имеют минимумы (в областях квазипересечений) вероятность неадиабатических переходов наиболее велика. В адиабатическом представлении этим областям соответствуют ненулевые матричные 
элементы неадиабатичности $\left\langle j\left|\frac{\partial}{\partial R}\right| k\right\rangle$ с экстремумами в областях квазипересечений потенциальных кривых. С вычислительной точки зрения данные экстремумы могут быть сложны в обработке. Это объясняется тем, что характерная ширина каждого $\delta$-образного пика оказывается сравнима, либо много меньше выбранного шага сетки интегрирования системы связанных уравнений ядерной подсистемы $[3,4]$. Дополнительно отметим, что такие пики могут быть существенно у́же самой области сближения потенциальных кривых.

Для устранения выявленных вычислительных трудностей предлагается сделать переход к другому диабатическому представлению операторов, входящих в систему связанных уравнений. При этом стремятся найти такое диабатическое представление, при котором матричные элементы неадиабатичности $\left\langle j\left|\frac{\partial}{\partial R}\right| k\right\rangle$ обнуляются в местах квазипересечений диагональных элементов электронного гамильтониана или претерпевают значительное уменьшение. Соответствующие потенциалы на главной диагонали матрицы электронного гамильтониана, как правило, претерпевают пересечения в этих областях межъядерных расстояний. Последнее дает более гладкие потенциальные кривые.

Базовая процедура диабатизации описана, в работе Смита [12] и в работе [13]. В случае молекулярных состояний с одинаковой симметрией она заключается в обнулении радиальных матричных элементов $\left\langle j\left|\frac{\partial}{\partial R}\right| k\right\rangle$ в целевом представлении. Для базовой процедуры существуют два основных требования. Одно из них заключается в равенстве нулю матричных элементов оператора $\frac{\partial}{\partial R}$ в диабатическом представлении на асимптотике. В реальных ситуациях это не всегда выполнимо, поскольку в молекулярном базисе некоторые из матричных элементов неадиабатичности могут оставаться ненулевыми в асимптотической области. Эта задача рассмотрена в работах $[14,15]$ и выходит за рамки обсуждаемой темы. Другое важное требование - это пересечение диабатических потенциальных кривых в областях неадиабатичности. Оба требования могут быть выполнены в случае полного набора молекулярных состояний [12].

Равенство нулю матричных элементов $\left\langle j\left|\frac{\partial}{\partial R}\right| k\right\rangle$ при всех значениях межъядерных расстояний составляет изначальное определение строгого диабатического представления, данное еще в работе Смита [12]. В общем случае переход к такому представлению является трудновыполнимым даже для двухатомных молекул [16], учитывая ограничение числа молекулярных термов в реальных задачах. Поэтому обнуление матричных элементов неадиабатичности обычно осуществляют не полностью и не при всех межъядерных расстояниях. Соответстующие представления называют квазидиабатическими, гибридными или просто диабатическими представлениями $[12,17]$.

Минимизация матричных элементов неадиабатичности составляет одну из стратегий диабатизации [18]. В рамках этой стратегии выделяется ряд методов, представленных, в частности, в работах [19-22]. В них определяются матрицы смены представления размерностью
$2 \times 2$ для каждой отдельно рассматриваемой области неадиабатичности. В работах [20-22] каждая матрица $2 \times 2$ определена как матрица вращения и ищется варьированием введенных в нее параметров. Указанные выше методы обеспечивают приближенное выполнение требований к процедуре диабатизации в выбранных областях неадиабатичности.

Конкретно в настоящей работе представлен метод диабатизации, который позволяет проводить выборочную минимизацию матричных элементов неадиабатичности $\left\langle j\left|\frac{\partial}{\partial R}\right| k\right\rangle$ путем приближения их кривыми Лоренца в рамках модели Ландау-Зинера. Предлагаемый метод более прост по сравнению с методами варьирования парамеров матрицы вращения, а также надежно обеспечивает выполнение двух основных требований к процедуре диабатизации для случая высокого и узкого изолированного пика матричного элемента неадиабатичности. Показано, что найденная на основе модельного матричного элемента Ландау-Зинера матрица смены представления $2 \times 2$ позволяет практически обнулить исходный матричный элемент $\left\langle j\left|\frac{\partial}{\partial R}\right| k\right\rangle$ и получить пересечение диабатических потенциальных кривых в заданной области.

Будет рассмотрена столкновительная система СаH, отличительной особенностью которой является наличие нескольких $\delta$-образных пиков в матричных элементах неадиабатичности. В частности, в работе рассматривается матричный элемент $\left\langle 8\left|\frac{\partial}{\partial R}\right| 9\right\rangle$. При межъядерном расстоянии 29.6340322 a.u. указанный матричный элемент имеет узкий пик высотой $1.227 \cdot 10^{4}$ a.u. с характерной шириной $8.1495 \cdot 10^{-5}$ a.u. Его местоположение совпадает с расстоянием наибольшего сближения адиабатических потенциалов $U_{8}$ и $U_{9}$.

Показано, что применение предложенного гибридного метода диабатизации на примере системы СаН позволило выполнить оба требования к процедуре для матричного элемента $\left\langle 8\left|\frac{\partial}{\partial R}\right| 9\right\rangle$ и одновременно устранить проблему его $\delta$-образного пика.

\section{2. Система связанных уравнений в решении задачи ядерной динамики}

Кратко изложим общий вывод системы связанных уравнений [4] для ядерной подсистемы в рамках подхода Борна-Оппенгеймера. Полный гамильтониан $\hat{H}$ всех частиц, составляющих столкновительную систему двух атомов имеет вид (в атомной системе единиц)

$$
\hat{H}=-\frac{1}{2 M} \Delta_{\mathbf{R}}-\sum_{\beta=1}^{N_{e}} \frac{1}{2 m_{\beta}} \Delta_{\mathbf{r}_{\beta}}+\hat{H}_{\text {int }}\left(\left\{\mathbf{r}_{\beta}\right\}, \mathbf{R}\right),
$$

где оператор Лапласа $\Delta_{\mathbf{R}}$ описывает движение ядер, $\Delta_{\mathbf{r}_{\beta}}$ соответствует движениям электронов в данной столкновительной системе, $\hat{H}_{\text {int }}\left(\left\{\mathbf{r}_{\beta}\right\}, \mathbf{R}\right)-$ оператор взаимодействий всех частиц в системе, $M$ - приведенная масса 
ядер, $m_{\beta}$ - последовательно вычисленные приведенные массы электронов, $\mathbf{R}(R, \Theta, \Phi)$ - ядерная координата, $\left\{\mathbf{r}_{\beta}\right\}-$ координаты Якоби электронов, $N_{e}-$ число электронов. В полном гамильтониане $\hat{H}$ выделяют электронный гамильтониан

$$
\hat{H}_{e}=-\frac{1}{2 m_{e}} \sum_{\beta=1}^{N_{e}} \Delta_{\mathbf{r}_{\beta}}+\hat{H}_{\mathrm{int}}\left(\left\{\mathbf{r}_{\beta}\right\}, \mathbf{R}\right),
$$

в котором $m_{e}=1$ - масса электрона.

Полный гамильтониан (1) содержит приведенные массы электронов $m_{\beta}$, тогда как при решении задачи нахождения квантово-химических данных обычно используется электронный гамильтониан вида (2). Матричные элементы операторов $\Delta_{\mathbf{r}_{\beta}}$, возникающие в связи с разностями $1 / m_{\beta}-1 / m_{e}$, считаются малыми поправками и не учитываются. Кроме этого, авторы принимают при решении ядерной задачи начало отсчета всех активных электронов от центра масс ядер. Поправки, связанные с этим приближением, также считаются малыми и не рассматриваются в настоящей работе.

Оператор взаимодействий $\hat{H}_{\text {int }}\left(\left\{\mathbf{r}_{\beta}\right\}, \mathbf{R}\right)$ включает в себя кулоновские взаимодействия всех частиц, составляющих квазимолекулу. Другие типы взаимодействия, такие как спин-орбитальное, вносящие малые вклады в общую картину столкновения, не относятся к теме настоящей работы и поэтому опущены [23].

Нахождение квантово-химических данных составляет отдельную проблему. В частности, для квазимолекулы СаН они приведены в работе [9].

Для молекулярных состояний ${ }^{2} \Sigma^{+}$полная волновая функция системы $\Psi(\mathbf{r}, \mathbf{R})$, являющаяся решением уравнения Шредингера $\hat{H} \Psi=E \Psi$, может быть представлена в следующем стандартном виде:

$$
\Psi(\mathbf{r}, \mathbf{R})=Y_{J M}(\Theta, \Phi) \sum_{k} \frac{F_{k}(R)}{R}\left|\phi_{k}(\mathbf{r}, \mathbf{R})\right\rangle,
$$

в котором $Y_{J \widetilde{M}}(\Theta, \Phi)-$ сферические гармоники, заданные квантовыми числами $J$ полного момента молекулы и его проекции $\widetilde{M}$ на молекулярную ось, $\left|\phi_{k}(\mathbf{r}, \mathbf{R})\right\rangle-$ базисные электронные функции адиабатического представления. В адиабатическом базисе (2) принимает диагональный вид, $F_{k}(R)$ представляют собой ядерные волновые функции в каналах $k$.

После подстановки волновой функции (3) в уравнение Шредингера можно получить систему связанных уравнений $[3,4]$ :

$$
\begin{aligned}
& \left\{-\frac{1}{2 M} \frac{d^{2}}{d R^{2}}+\frac{J(J+1)}{2 M R^{2}}+\left(U_{j}(R)-E\right)\right\} F_{j} \\
& =\frac{1}{M} \sum_{k}\left\langle\phi_{j}\left|\frac{\partial}{\partial R}\right| \phi_{k}\right\rangle \frac{d}{d R} F_{k}+\frac{1}{2 M} \sum_{k}\left\langle\phi_{j}\left|\frac{\partial^{2}}{\partial R^{2}}\right| \phi_{k}\right\rangle F_{k} .
\end{aligned}
$$

В системе уравнений (4) $j=\overline{1 ; \infty}$ в общем случае, либо $j=\overline{1 ; N}$ в приближении связанных каналов, где $N-$ некоторое конечное целое число, $k$ меняется так же, как и $j$. Потенциалы $U_{j}(R)=\left\langle\phi_{j}\left|\hat{H}_{e}\right| \phi_{j}\right\rangle$ определены, как это непосредственно видно, в виде диагональных матричных элементов электронного гамильтониана в представлении с адиабатическим базисом $\left\{\left|\phi_{j}\right\rangle\right\}$. Матричные элементы $\left\langle\phi_{j}\left|\frac{\partial}{\partial R}\right| \phi_{k}\right\rangle$ называются матричными элементами неадиабатичности и в зависимости от выбранного представления могут в той или иной степени отвечать за вероятность неадиабатических переходов. В адиабатическом представлении данные матричные элементы полностью определяют указанные вероятности. В некоторых случаях свой вклад также вносят и матричные элементы $\left\langle\phi_{j}\left|\frac{\partial^{2}}{\partial R^{2}}\right| \phi_{k}\right\rangle$.

\section{1. Смена представления в системе связанных уравнений}

Адиабатическое представление дает наглядность описания эволюции столкновительной системы как квазимолекулы. Однако его использование встречает вычислительные неудобства при наличии матричных элементов неадиабатичности $\left\langle\phi_{j}\left|\frac{\partial}{\partial R}\right| \phi_{k}\right\rangle$, имеющих острые пики. Примером этому служит одна из областей неадиабатичности для матричного элемента $\left\langle 8\left|\frac{\partial}{\partial R}\right| 9\right\rangle$ в приближении ограниченного числа ${ }^{2} \Sigma^{+}$-состояний столкновительной системы СаН. Традиционно такие пики устраняются путем смены представления операторов, входящих в систему уравнений (4). В новом диабатическом представлении с базисом $\left\{\left|\chi_{j}\right\rangle\right\}$ эта система связанных уравнений принимает вид

$$
\begin{aligned}
& \left\{-\frac{1}{2 M} \frac{d^{2}}{d R^{2}}+\frac{J(J+1)}{2 M R^{2}}-E\right\} F_{j} \\
& =\frac{1}{M} \sum_{k}\left\langle\chi_{j}\left|\frac{\partial}{\partial R}\right| \chi_{k}\right\rangle \frac{d}{d R} F_{k} \\
& \quad+\frac{1}{2 M} \sum_{k}\left\langle\chi_{j}\left|\frac{\partial^{2}}{\partial R^{2}}\right| \chi_{k}\right\rangle F_{k}-\sum_{k}\left\langle\chi_{j}\left|\hat{H}_{e}\right| \chi_{k}\right\rangle F_{k} .
\end{aligned}
$$

В уравнении (5) сумма по матричным элементам гамильтониана включает диабатические потенциалы. В матричной записи элементы операторов систем (4) и (5) связаны соотношениями

$$
\begin{gathered}
P_{\chi}=C^{+} P_{\phi} C+C^{+} \frac{d}{d R} C=C^{+}\left(P_{\phi} C+\frac{d}{d R} C\right), \\
P_{\chi}^{(2)}=C^{+} P_{\phi}^{(2)} C+C^{+} \frac{d^{2}}{d R^{2}} C+2 C^{+} P_{\phi} \frac{d}{d R} C, \\
H_{\chi}=C^{+} H_{\phi} C,
\end{gathered}
$$

где $P_{\chi}=\left\langle\chi_{j}\left|\frac{\partial}{\partial R}\right| \chi_{k}\right\rangle, P_{\phi}=\left\langle\phi_{j}\left|\frac{\partial}{\partial R}\right| \phi_{k}\right\rangle, P_{\chi}^{(2)}=\left\langle\chi_{j}\left|\frac{\partial^{2}}{\partial R^{2}}\right| \chi_{k}\right\rangle$, $P_{\phi}^{(2)}=\left\langle\phi_{j}\left|\frac{\partial^{2}}{\partial R^{2}}\right| \phi_{k}\right\rangle, H_{\chi}$ - матрица гамильтониана в диабатическом базисе, $H_{\phi}-$ матрица гамильтониана в адиабатическом базисе, $C$ - матрица унитарного преобразования. 
Выражение в скобках в формуле (6) является ключевым для общепринятого подхода к диабатизации, указанного в работе Смита [12]. Согласно ему новое диабатическое представление выбирается таким, чтобы элементы $P_{\chi}$ были равны нулю. Тогда можно сократить вынесенную за скобки матрицу $C^{+}$и получить систему дифференциальных уравнений первого порядка по $R$ для элементов матрицы $C$ :

$$
\frac{d}{d R} C=-P_{\phi} C
$$

Решение этой системы определяется граничным условием $C_{0}=I$ при достаточно большом межъядерном расстоянии $R_{\infty}$. Решение системы (9) производится от $R_{\infty}$ в сторону $R=0$.

В случае отдельно взятых острых пиков в матричных элементах неадиабатичности данная система уравнений может решаться в интервалах локализации каждого такого пика. В разд. 2.2 и 2.3 изложен предлагаемый авторами метод перехода к гибридному диабатическому представлению, осуществляемому в интервале локализации острых пиков матричных элементов $\left\langle\phi_{j}\left|\frac{\partial}{\partial R}\right| \phi_{k}\right\rangle$, который позволил получить удобные для проведения вычислений квантово-химические данные.

\section{2. Использование модельных матричных элементов неадиабатичности для нахождения гибридного диабатического представления}

Основной целью предлагаемой процедуры диабатизации является гарантированность пересечения диабатических потенциалов в области квазипересечения адиабатических потенциальных кривых. Для выполнения данного требования авторы предлагают представлять исходные матричные элементы $P_{\phi}$ в виде суммы

$$
P_{\phi}=P^{m}+P^{\text {res }},
$$

где $P^{m}$ - модельные матричные элементы неадиабатичности, имеющие известный аналитический вид и обеспечивающие пересечение выбранных диабатических потенциалов, а $P^{\text {res }}$ - остаток от исходных матричных элементов $P_{\phi}$. Остаток $P^{\text {res }}$ считается значительно меньшим по сравнению с $P^{m}$.

Допустим на время, что наши исходные матричные элементы неадиабатичности $P_{\phi}$ равны модельным $P^{m}$ и стандартная процедура диабатизации по Смиту с такими матричными элементами обеспечивает пересечение диабатических потенциалов в представлении $\chi$. Тогда система уравнений (9) относительно модельных матричных элементов будет представлена в виде

$$
\frac{d}{d R} C=-P^{m} C
$$

Заменим производную $\frac{d}{d R} C$ в (6) ее выражением через $P^{m}$ из матричного уравнения (11). Такая подстановка приводит к равенству

$$
\begin{aligned}
P_{\chi} & =C^{+}\left(P_{\phi} C+\frac{d}{d R} C\right)=C^{+}\left(P_{\phi} C-P^{m} C\right)= \\
& =C^{+}\left(P_{\phi}-P^{m}\right) C=C^{+} P^{\text {res }} C=P^{\text {res }} .
\end{aligned}
$$

Из выражения (12) видно, что представление матричных элементов $P_{\phi}$ в виде суммы известных модельных элементов $P^{m}$ и малых остаточных элементов $P^{\text {res }}$ дает новые элементы $P_{\chi}$ в диабатическом представлении, которые равны $P^{\text {res }}$. Одновременно с этим мы исходили из того, что матрица $C$, найденная при решении системы уравнений (11) относительно элементов $P^{m}$ некоторой аналитической модели описания областей неадиабатичности, гарантирует пересечение диабатических потенциальных кривых. Следовательно, можно выбрать модельные матричные элементы неадиабатичности, которые позволят значительно уменьшить узкие пики матричных элементов $\left\langle\phi_{j}\left|\frac{\partial}{\partial R}\right| \phi_{k}\right\rangle$, заменив их остаточными функциями. Кроме того, каждый модельный матричный элемент неадиабатичности должен убывать до нуля при удалении от области своего пика. Это позволяет избежать учета постоянных ненулевых матричных элементов при нахождении матрицы пребразования $C$.

\section{3. Применение модели Ландау-Зинера в процедуре избирательной диабатизации матричных элементов неадиабатичности}

Хорошо известной моделью, при которой диабатические потенциалы имеют пересечение в области неадиабатичности, является модель Ландау-Зинера. Ее изложение можно найти в [24]. Применение этой модели к задаче вычисления сечений возбуждения также изложено в [25]. Согласно модели Ландау-Зинера, элемент неадиабатической связи между двумя соседними адиабатическими потенциалами задается формулой

$$
\begin{aligned}
& P_{j k}^{L Z}(R)=\frac{\tau}{\left(R-R_{0}\right)^{2}+4 \tau^{2}}, \\
& \tau=\frac{H_{j k}}{\left|d H_{j j} / d R-d H_{k k} / d R\right|},
\end{aligned}
$$

где элементы $H_{j k}, H_{j j}$ и $H_{k k}$ представляют собой часть матрицы гамильтониана для двух соседних каналов $j$ и $k$ в диабатическом представлении.

Предлагаемая авторами процедура диабатизации заключается в решении системы уравнений (11) с модельными матричными элементами (13) для выбранных областей квазипересечений адиабатических потенциалов с одиночными высокими и узкими пиками матричных элементов неадиабатичности. Параметры модельных кривых $\tau$ и $R_{0}$ из формулы (13) должны оптимальным образом приближать исходные $\delta$-образные пики матричных элементов неадиабатичности из входных квантовохимических данных. Эти два параметра находятся по высоте и положению экстремумов исходных таблиц 
Таблица 1. Молекулярные состояния $\mathrm{CaH}\left({ }^{2} \Sigma^{+}\right)$, соответствующие асимптотические атомные состояния и асимптотические энергии $[9,27]$ относительно основного состояния

\begin{tabular}{c|c|c}
\hline$j$ & $\begin{array}{c}\text { Асимптотические } \\
\text { атомные состояния }\end{array}$ & $\begin{array}{c}\text { Асимптотическая } \\
\text { энергия, } \mathrm{eV}\end{array}$ \\
\hline 1 & $\mathrm{Ca}\left(4 s^{2}{ }^{1} S\right)+\mathrm{H}\left(1 s^{2} S\right)$ & 0.0 \\
2 & $\mathrm{Ca}\left(4 s 4 p^{3} P\right)+\mathrm{H}\left(1 s^{2} S\right)$ & 1.892 \\
3 & $\mathrm{Ca}\left(3 d 4 s^{3} D\right)+\mathrm{H}\left(1 s^{2} S\right)$ & 2.524 \\
4 & $\mathrm{Ca}\left(3 d 4 s^{1} D\right)+\mathrm{H}\left(1 s^{2} S\right)$ & 2.709 \\
5 & $\mathrm{Ca}\left(4 s 4 p^{1} P\right)+\mathrm{H}\left(1 s^{2} S\right)$ & 2.933 \\
6 & $\mathrm{Ca}\left(4 s 5 s^{3} S\right)+\mathrm{H}\left(1 s^{2} S\right)$ & 3.910 \\
7 & $\mathrm{Ca}\left(4 s 5 s^{1} S\right)+\mathrm{H}\left(1 s^{2} S\right)$ & 4.131 \\
8 & $\mathrm{Ca}\left(3 d 4 p^{3} F\right)+\mathrm{H}\left(1 s^{2} S\right)$ & 4.442 \\
9 & $\mathrm{Ca}\left(4 s 5 p^{3} P\right)+\mathrm{H}\left(1 s^{2} S\right)$ & 4.534 \\
10 & $\mathrm{Ca}\left(4 s 5 p^{1} P\right)+\mathrm{H}\left(1 s^{2} S\right)$ & 4.554 \\
11 & $\mathrm{Ca}+\left(4 s^{2} S\right)+\mathrm{H}-\left(1 s^{2} S\right)$ & 5.363
\end{tabular}

Таблица 2. $\delta$-образные матричные элементы неадиабатичности из набора квантово-химических данных квазимолекулы $\mathrm{CaH}$

\begin{tabular}{c|c|c|c}
\hline$\left\langle j\left|\frac{\partial}{\partial R}\right| k\right\rangle$ & $R_{0}$, a.u. & $\left\langle j\left|\frac{\partial}{\partial R}\right| k\right\rangle\left(R_{0}\right)$, a.u. & $\begin{array}{c}\text { Ширина пика } \\
\text { полувысоте, } \\
\text { а.u. }\end{array}$ \\
\hline$\left\langle 8\left|\frac{\partial}{\partial R}\right| 9\right\rangle$ & 29.6340322 & $1.227 \cdot 10^{4}$ & $8.1495 \cdot 10^{-5}$ \\
$\left\langle 8\left|\frac{\partial}{\partial R}\right| 9\right\rangle$ & 9.7788768 & 46.18 & $2.1655 \cdot 10^{-2}$ \\
$\left\langle 8\left|\frac{\partial}{\partial R}\right| 9\right\rangle$ & 6.2800250 & 18.92 & $5.286 \cdot 10^{-2}$ \\
$\left\langle 4\left|\frac{\partial}{\partial R}\right| 5\right\rangle$ & 5.2377523 & -32.85 & $3.044 \cdot 10^{-2}$ \\
$\left\langle 10\left|\frac{\partial}{\partial R}\right| 11\right\rangle$ & 4.4895217 & 96.87 & $1.032 \cdot 10^{-2}$
\end{tabular}

функций матричных элементов неадиабатичности [26]. Найденная на основе таких модельных матричных элементов матрица преобразования $C$ приводит к пересечению диабатических потенциалов. Убывание модельных матричных элементов неадиабатичности (11) до значений, близких к нулю, при отдалении от области неадиабатичности, позволяет избежать решения проблемы постоянных ненулевых матричных элементов в рамках процедуры диабатизации. Для тех областей неадиабатичности, где диабатизацию проводить не нужно, модельные матричные элементы можно положить равными нулю.

Таким образом, получаемая матрица преобразования $C$ позволяет переходить к гибридному диабатическому представлению. В этом представлении присутствуют остаточные матричные элементы неадиабатичности $P^{\text {res }}$, соответствующие им недиагональные элементы электронного гамильтониана и часть исходных матричных элементов из $P_{\phi}$.

Приближение узких пиков матричных элементов неадиабатичности функциями (13) в рамках модели Ландау-Зинера позволяет добиться значительного уменьшения матричного элемента неадиабатичности и гарантированного пересечения в изолированной области неадиабатичности для двух потенциалов. Изложенный подход ограничен случаями непересекающихся областей неадиабатичности для выбранных пар состояний. Случай трех и более соседних состояний и пересекающихся соседних областей неадиабаичности на данном этапе не рассматривается.

В следующем разделе будут изложены результаты диабатизации в выбранной области неадиабатичности для системы $\mathrm{CaH}$ для состояний 8 и 9. Именно эта область неадиабатичности вызвала наибольшие трудности для решения задачи ядерной динамики в рамках квантового подхода.

\section{4. Выборочная диабатизация данных для системы $\mathrm{CaH}$}

В процедуре диабатизации авторами использованы квантово-химические данные для первых одиннадцати ${ }^{2} \Sigma^{+}$-каналов квазимолекулы СаН, взятые из работы [9]. В табл. 1 даны асимптотические энергии данной квазимолекулы, полученные в работе [9] на основе [27].

Анализ квантово-химических данных для столкновительной системы СаН показал наличие нескольких областей неадиабатичности, в которых необходимо провести диабатизацию. Матричные элементы неадиабатичности в этих областях имеют форму узких пиков, что отражено в табл. 2.

Гибридные потенциальные энергии, полученные в результате предложенной в данной работе процедуры диабатизации, представлены на рис. 1.

Наиболее показательной является область неадиабатичности между состояниями 8 и 9 в районе

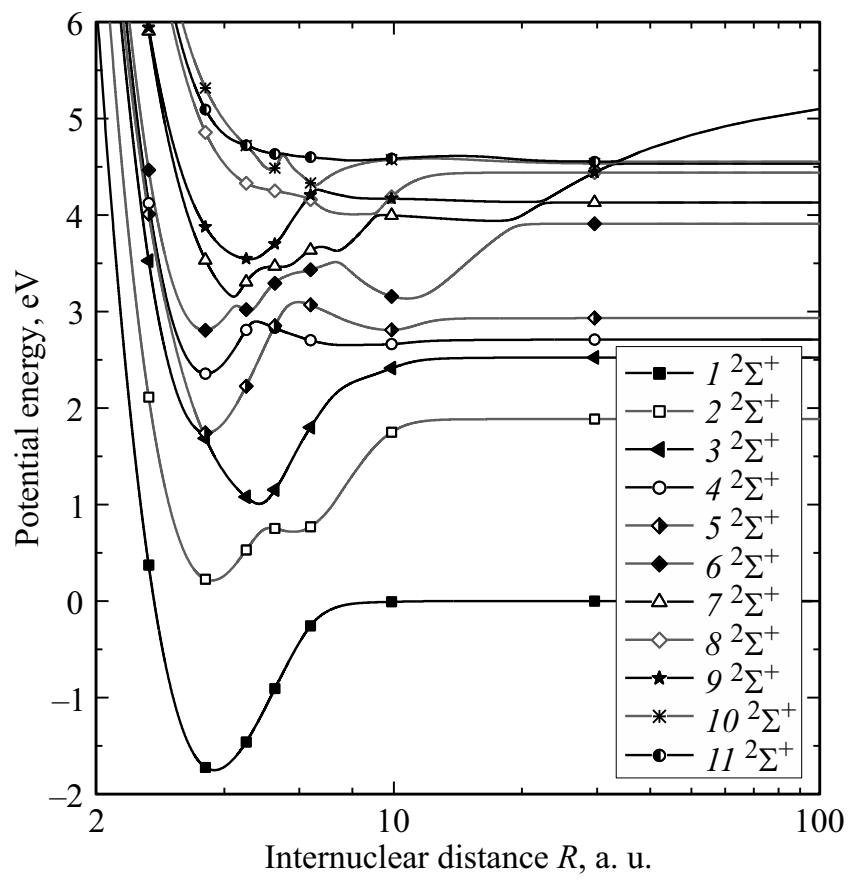

Рис. 1. Квантово-химические данные. Гибридные потенциальные энергии $\mathrm{CaH}\left({ }^{2} \Sigma^{+}\right)$в зависимости от межъядерного расстояния $R$ (расшифровка состояний представлена в табл. 1). 


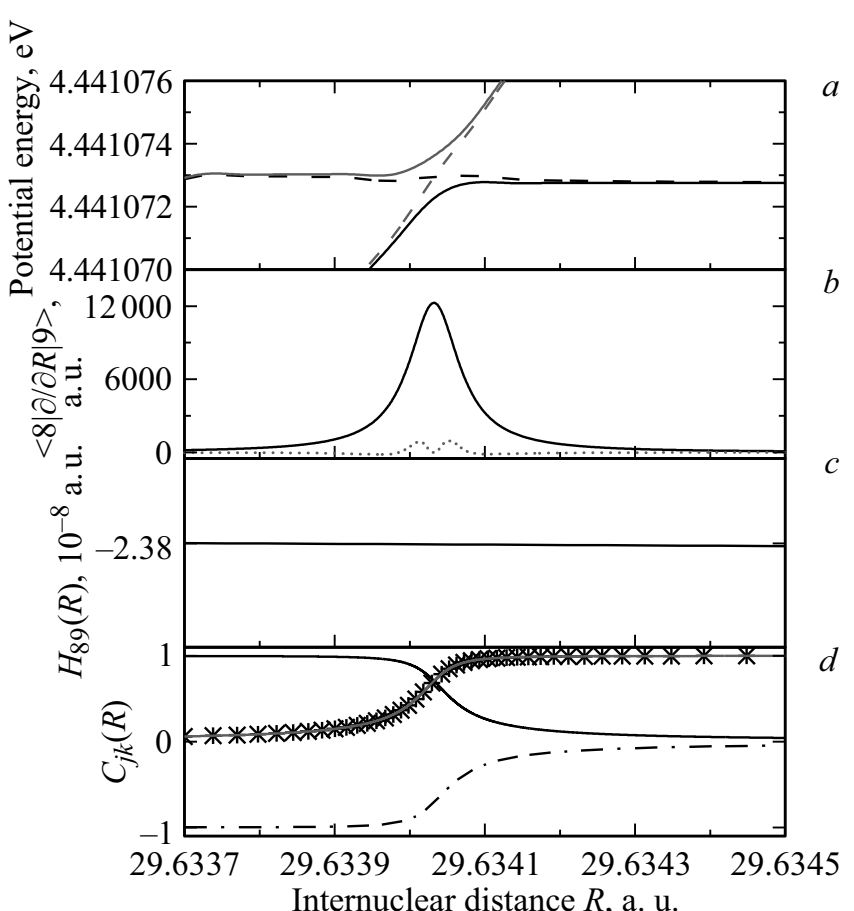

Рис. 2. Квантово-химические данные: ( $a$ ) потенциальные энергии в адиабатическом $U_{8}$ (черная сплошная кривая) и $U_{9}$ (серая сплошная кривая) и диабатическом $H_{88}$ (черная штриховая кривая) и $H_{99}$ (серая штриховая кривая) представлениях; (b) матричные элементы неадиабатического взаимодействия $\langle 8|\partial / \partial R| 9\rangle$ : приближенный модельный матричный элемент (сплошная линия) и остаточный элемент, увеличенный в 100 раз (точечная кривая); (c) недиагональный матричный элемент $H_{89} ;(d)$ элементы матрицы смены представления $C_{88}$ (символы), $C_{89}$ (черная сплошная линия), $C_{98}$ (штрихпунктир), $C_{99}$ (серая сплошная линия) в зависимости от межъядерного расстояния $R$.

$R_{0} \approx 29.6340322$ a.u. Квантово-химические данные в адиабатическом представлении для этой области неадиабатичности также взяты из работы [9]. В этой области матричный элемент неадиабатичности $\left\langle 8\left|\frac{\partial}{\partial R}\right| 9\right\rangle$ имеет узкий пик высотой $1.227 \cdot 10^{4}$ a.u. и шириной $\approx 8.1495 \cdot 10^{-5}$ а.и.. Соответствующее значение расщепления потенциалов $\left|U_{9}\left(R_{0}\right)-U_{8}\left(R_{0}\right)\right| \approx 5 \cdot 10^{-8}$ a.u. Рис. 2 содержит результаты диабатизации этой части квантово-химических данных. На рис. 2, а можно видеть пересечение диабатических потенциалов $H_{88}$ и $H_{99}$ в точке сближения адиабатических потенциальных кривых $U_{8}$ и $U_{9}$. Рис. $2, b$ содержит уже приближенный модельный элемент $\left\langle 8\left|\frac{\partial}{\partial R}\right| 9\right\rangle^{L Z}$, который фактически совпадает с исходным матричным элементом неадиабатичности. На этом же рисунке изображен график остаточного матричного элемента неадиабатичности $\left\langle 8\left|\frac{\partial}{\partial R}\right| 9\right\rangle^{\text {res }}$ (точечная кривая), рассчитанного по формуле (12). Этот последний матричный элемент намеренно умножен на 100 всюду в интервале графика, поскольку его сравнение с высотой модельного и входного матричных элементов неадиабатичности визуально затруднительно. Недиаго- нальный элемент гамильтониана $H_{89}$, представленный на рис. 2,c, является малой постоянной, что согласуется с моделью Ландау-Зинера. Последний рис. 2, $d$ показывает зависимость от межъядерного расстояния подматрицы $2 \times 2$ преобразования $C$ для рассматриваемых каналов. Она представляет собой вещественную ортогональную матрицу - матрицу вращения. В данном конкретном случае эта матрица получена численным путем без использования ее аналитического вида [12]. Для более чем двух соседних состояний и пересекающихся областей неадиабатичности процедура диабатизации на данном этапе исследования авторами не рассматривалась.

\section{Заключение}

Авторами представлен гибридный метод диабатизации изолированных областей неадиабатичности для $\delta$ образных пиков матричных элементов $\left\langle j\left|\frac{\partial}{\partial R}\right| k\right\rangle$ с вычислением матрицы смены представления относительно кусочно-приближенных матричных элементов неадиабатичности по модели Ландау-Зинера. Это позволило получить гарантированное пересечение диабатических потенциальных кривых и выборочно устранить указанные узкие $\delta$-образные пики в матричных элементах неадиабатичности в гибридном диабатическом представлении. Конкретно, для квантово-химических данных одиннадцати каналов ${ }^{2} \Sigma^{+}$для столкновительной системы СаН были диабатизированы участки с $\delta$-образными матричными элементами неадиабатичности из областей межъядерных расстояний, указанных в табл. 2.

Для остальных областей неадиабатичности используются исходные данные адиабатического представления с точностью до знаков матричных элементов неадиабатичности, получаемых на выходе после диабатизации выбранных областей неадиабатичности. Элементы матрицы преобразования могут приводить к перестановкам и смене знаков матричных элементов неадиабатичности всех затронутых каналов.

Работа поддержана Российским научным фондом, грант № 17-13-01144.

\section{Список литературы}

[1] Машонкина Л.И., Беляев А.К., Ши Ж.-Р. // Письма в Астрономический журнал. 2016. Т. 42. № 6. С. 408. doi 10.7868/S0320010816050077; Mashonkina L.I., Belyaev A.K., Shi J.-R. // Astronomy Letters. 2016. V. 42. N 6. P. 366. doi 10.1134/S1063773716050078

[2] Born M., Oppenheimer J. R. // Annals of Physics. 1927. V. 84. P. 457.

[3] Belyaev A.K. // Eur. Phys. J. D. 2007. V. 44. P. 497. doi 10.1140/epjd/e2007-00203-9

[4] Belyaev A.K. // Russ. J. Phys. Chem. A. 2002. V. 76. Suppl. 1, P. 68.

[5] Belyaev A.K., Barklem P.S. // Phys. Rev. A. 2003. V. 68, P. 062703. doi 10.1103/PhysRevA.68.062703

[6] Belyaev A.K., Grosser J., Hahne J., Menzel T. // Phys. Rev. A. 1999. V. 60. P. 2151. doi 10.1103/PhysRevA.60.2151 
[7] Власов Д.В., Барклем П.С., Беляев А.К. // Опт. и спектр. 2011. T. 110. № 3. C. 355; Vlasov D.V., Barklem P.S., Belyaev A.K. // Opt. Spectr. 2011. V. 110. N 3. P. 321. doi 10.1134/S0030400X1103026X

[8] Rodionov D.S., Belyaev A.K., Guitou M., Spielfiedel A., Feautrier N., Barklem P.S. // Izvestia of Herzen University. 2014. V. 165. P. 63.

[9] Mitrushchenkov A., Guitou M., Belyaev A.K., Yakovleva S.A., Spielfiedel A., Feautrier N. // J. Chem. Phys. 2017. V. 146. P. 014304. doi 10.1063/1.4973457

[10] Belyaev A.K., Yakovleva S.A., Guitou M., Mitrushchenkov A.O., Spielfiedel A., Feautrier N. // Astron. Astrophys. 2016. V. 587. P. A114. doi 10.1051/0004-6361/201527651

[11] Belyaev A.K., Barklem P.S., Dickinson A.S., Gadéa F.X. // Phys. Rev. A. 2010. V. 81. P. 032706. doi 10.1103/PhysRevA.81.032706

[12] Smith F. // Phys. Rev. 1969. V. 179. P. 111. doi 10.1103/PhysRev.179.111

[13] Worth G.A., Cederbaum L.S. // Annu. Rev. Phys. Chem. 2004. V. 55. P. 127. doi 10.1146/annurev.physchem.55.091602.094335

[14] Belyaev A. K. // Phys. Rev. A. 2010. V. 82. P. 060701. doi 10.1103/PhysRevA.82.060701

[15] Беляев А.К., Власов Д.В., Касьянова А.М. // Опт. и спектр. 2007. T. 103. № 6. C. 956; Belyaev A.K., Vlasov D.V., Kas'yanova A.M. // Opt. Spectr. 2007. V. 103. N 6. P. 920. doi 10.1134/S0030400X07120144

[16] Mead C.A., Truhlar D.G. // J. Chem. Phys. 1982. V. 77. P. 6090. doi 10.1063/1.443853

[17] Garrett B.C., Truhlar D.G., Melius C.F. Derivative Coupling Elements in Electronically Adiabatic Representations and Their Use in Scattering Calculations. Energy Storage and Redistribution in Molecules. NY.: Plenum Press, 1983. P. 375.

[18] Van Voorhis T., Kowalczyk T., Kaduk B., Wang L.-P., Cheng C.-L., Wu Q. // Annu. Rev. Phys. Chem. 2010. V. 61. P. 149. doi 10.1146/annurev.physchem.012809.103324

[19] Mitrushenkov A.O., Palmieri P., Puzzarini C., Tarroni R. // Mol. Phys. 2000. V. 98. P. 1677. doi 10.1080/00268970009483373

[20] Zanchet A., Bañares L., Senent M. L., García-Vela A. // Phys. Chem. Chem. Phys. 2016. V. 18. P. 33195. doi $10.1039 / \mathrm{c} 6 \mathrm{cp} 05960 \mathrm{k}$

[21] Roos J.B., Orel A.E., Larson Å. // Phys. Rev. A. 2009. V. 79. P. 062510. doi 10.1103/PhysRevA.79.062510

[22] Roos J.B., Larsson M., Larson Å, Orel A.E. // Phys. Rev. A. 2009. V. 80. P. 012501. doi 10.1103/PhysRevA.80.012501

[23] Truhlar D.G. Potential Energy Surfaces. Encyclopedia of Physical Science and Technology (Third Edition). N.Y.: Academic Press, 2001. V. 13. P. 9.

[24] Никитин Е.Е., Уманский С.Я. Неадиабатические переходы при медленных атомных столкновениях. М.: Атомиздат, 1979. 272 c.; Nikitin E.E., Umanskii S.Y. Theory of Slow Atomic Collisions. Berlin: Springer-Verlag, 1984. 444 p.

[25] Беляев А.К. // Известия РГПУ им. А.И. Герцена. 2006. Т. 6. C. 213.

[26] Villaverde K., Kreinovich V. // Interval Computations. 1993, N 4. P. 176.

[27] Kramida A., Ralchenko Y., Reader J. and NIST ASD Team. NIST Atomic Spectroscopy Database, Version 5.0. Gaithersburg: National Institute of Standards and Technology, 2012. [Электронный ресурс] Режим доступа: http://physics.nist.gov/asd 